\title{
Estrategias de aprendizaje y adquisición de una segunda lengua
}

\author{
Antonio Fernández-Castillo. Universidad de Granada
}

Recepción: 28 de diciembre de 2015 | Revisión: 28 de diciembre de 2015 | Aceptación/Publicación: 29 de diciembre de 2015 Correspondencia: afcastil@ugr.es

Citar: Fernández-Castillo, A. (2016). Estrategias de aprendizaje y adquisición de una segunda lengua. ReiDoCrea, 4, $491-404$.

\begin{abstract}
Resumen: Las estrategias de aprendizaje involucradas en la adquisición de una segunda lengua han sido un tema de alto interés en los últimos años para diferentes áreas de la investigación Psico-educativa, sin que por el momento haya consenso sobre su categorización, el carácter de su funcionamiento o sobre su potenciación en el contexto aplicado. El objetivo de este trabajo es presentar una revisión sobre el estado actual de este tema. Para ello se lleva a cabo un análisis conceptual sobre la noción de estrategia de aprendizaje, sus tipologías y sus posibilidades de clasificación, además de sus implicaciones a la luz de lo que el conocimiento psicopedagógico actual permite ofrecer. Se realiza un análisis descriptivo y conceptual de las dos grandes categorías generalmente reconocidas de estrategias de aprendizaje: primarias y secundarias, revisando las tipologías recogidas en cada uno de estos conjuntos. Por último se lleva a cabo un análisis de algunas de las implicaciones aplicadas y posibilidades de intervención.
\end{abstract}

Palabras clave: Enseñanza de una Segunda Lengua I Estrategias Educativas

Learning Strategies and Second Language Acquisition

\begin{abstract}
Learning strategies involved in the acquisition of a second language have been a subject of great interest in recent years for different areas of psycho-educational research, although at present there is no consensus on the categorization, the nature of their operation or their use in the applied context. The aim of this paper is to present a review of the current status of this topic. To this end a conceptual analysis of the concept of learning strategy, its types and the possibilities of classification, as well as the implications in the light of what the current psycho-pedagogical knowledge offers are carried out. A descriptive and conceptual analysis of the two generally recognized categories of learning strategies: primary and secondary is carried out, reviewing the types contained in each of these sets. Finally an analysis of some of the applied implications and possibilities for intervention is carried out.
\end{abstract}

Key Words: Second Language Instruction I Educational Strategies

\section{Introducción}

Desde hace tiempo y desde posicionamientos teóricos considerados hoy en día clásicos, se ha reconocido, entre otros factores, la importancia de varios elementos de alta incidencia en todo proceso reglado de enseñanza-aprendizaje (Castejón, 2014; Weinstein y Mayer, 1986). Todos ellos podrían clasificarse en seis conjuntos que recordamos a continuación.

En un primer apartado se suele incluir frecuentemente al profesor y sus características personales, su experiencia, conocimientos y formación, habilidades y personalidad, accesibilidad, etc. En segundo lugar se encontraría el discente. En este elemento de análisis se incluyen una amplia lista de variables relevantes, tales como sus características personales, motivación, aptitudes y actitudes hacia el aprendizaje específico, conocimientos previos y experiencia en el área concreta, destrezas, etc. En tercer lugar frecuentemente se consideran las estrategias docentes, donde se ubicaría la metodología docente, incluyendo las actividades y procesos diseñados y puestos en marcha para el trabajo en el proceso. En cuarto lugar se encontraría el contexto y ambiente de aprendizaje. Se considerarían aquí aquellos elementos que inciden en los procesos de enseñanza y que los afectan tanto positiva como negativamente. Han sido ampliamente estudiados aquí desde las relaciones entre iguales, los influjos sociales, las particularidades de los sistemas educativos, sus legislaciones, políticas, etc., hasta aspectos específicos de los centros educativos o familiares, entre otros. En quinto lugar habríamos de tener en cuenta los sistemas de evaluación. Este elemento 
se encontraría íntimamente relacionado con las estrategias docentes y la ejecución. Un sistema de evaluación sería cualquier procedimiento por el cual cualquier discente es capaz de demostrar tanto cualitativa como cuantitativamente, en qué medida ha alcanzado los objetivos de aprendizaje propuestos y las competencias previstas. Su importancia estriba en que no todos los sistemas, técnicas y procesos de evaluación permiten alcanzar los objetivos para los que fueron diseñados, variando significativamente en función de los elementos anteriores. En último lugar ubicaríamos el punto nuclear en el que nos centraremos a lo largo de este trabajo, las estrategias de aprendizaje. Este apartado incluye esencialmente el análisis de lo que hace un estudiante para alcanzar los objetivos de aprendizaje propuestos.

Estos seis conjuntos de variables han sido estudiados en el caso del aprendizaje de una segunda lengua (en adelante L2). De hecho en cualquier área de aprendizaje el papel que desempeñan y sus contribuciones se consideran condiciones necesarias e imprescindibles para que se produzca aprendizaje eficiente y así, en el estudio específico del desarrollo y adquisición de una L2, los aspectos que potencian y dificultan este proceso han suscitado desde hace tiempo el interés de investigadores y docentes.

Y en esta línea, las estrategias y los estilos de aprendizaje ha conformado una de las temáticas que ha llamado poderosamente la atención ya que su importancia desde una perspectiva aplicada, parece capital. De ahí que un área relativamente extensa en Psicología de la Educación se haya centrado en investigar cuales son los procesos cognitivos por los cuales un discente llega a alcanzar un aprendizaje determinado, cuales son las tareas y los pasos para llegar a esa meta (Rodríguez, Valle y Núñez, 2014). En el caso del aprendizaje de una L2, ha preocupado igualmente conocer lo que hacen los buenos discentes para poder aplicar esos procedimientos y estrategias a otros casos.

O en otras palabras, el interés estribaría en conocer qué hacen los buenos estudiantes para prender, de cara a conocer cómo podrían optimizarse estos procesos en aquellos casos donde el aprendizaje es menos efectivo o eficiente. Este encuadre básico se complejiza con preguntas que los investigadores se plantean: cuales son los procesos seguidos a lo largo del proceso de aprendizaje de una L2, los mecanismos cognitivos utilizados, la planificación desarrollada y sus pasos, etc. Diversas aproximaciones han intentado explorar por ejemplo, qué estrategias de los estudiantes de una segunda lengua se correlacionan con mejores resultados, expresados por ejemplo en la competencia alcanzada (Liu, 2004; Wu, 2008).

El estudio de las estrategias de aprendizaje que siguen los estudiantes, persigue en el fondo dar respuesta a una serie de interrogantes específicos a los que amplias áreas de la Psicología y la Pedagogía intentan encontrar respuesta. Entre estas preguntas, en el caso concreto del aprendizaje de una L2, se encuentran las siguientes. ¿Qué hace un estudiante de éxito cuando se enfrenta con el aprendizaje de una segunda lengua? ¿Qué hace realmente que un estudiante de idiomas destaque por encima de otros? ¿Qué variables interactúan e inciden en la puesta en práctica de las estrategias de aprendizaje específicas? ¿Cómo podemos optimizar el trabajo y el rendimiento de un estudiante en una situación de aprendizaje de una segunda lengua?

La respuesta a este planeamiento de investigación ha sido diversa y multifacética por la cantidad ingente de variables que se ven implicadas en tales procesos. Parece claro que los buenos estudiantes usan una variedad amplia de estrategias de aprendizaje, pero además lo hacen de manera apropiada, eficiente y aplicando principios de flexibilidad (Chamot, O'Malley, Kupper y Impink-Hernandez, 1998), dando cabida a 
procesos que priorizan la comprensión, la práctica y la memorización de contenidos relevantes (Takeuchi, 2003).

A lo largo de este trabajo nos planteamos como objetivos llevar a cabo una revisión sobre el estado actual de este tema. Para ello comenzaremos con una exploración del concepto de estrategia de aprendizaje, sus tipologías y sus posibilidades de clasificación, sus implicaciones a la luz de lo que el conocimiento psicopedagógico actual permite ofrecer, para, por último, llevar a cabo un análisis de algunas de las implicaciones aplicadas y posibilidades de intervención.

\section{Estrategias de aprendizaje: delimitación conceptual}

Del avance y la aproximación anterior al concepto de estrategias de aprendizaje podría pensarse que la labor de definición de este concepto es fácil, que está terminada o que hay consenso entre investigadores y profesionales sobre su delimitación. Nada más lejos de la realidad, las definiciones que podemos encontrar son múltiples variando ampliamente la aproximación teórica y estructural de su elaboración. De hecho no sólo existen diferencias entre las conceptualizaciones de este término, sino que incluso otros, tales como conductas de aprendizaje, tácticas, recursos, destrezas $\mathrm{u}$ otros, han sido entendidos en ocasiones como sinónimos al de estrategias de aprendizaje (Griffiths, 2008).

Algunas de las definiciones tradicionales procedentes del ámbito de la Psicología y la Psicología de la Educación, partían de conceptualizaciones del funcionamiento cognitivo humano que a su vez diferenciaba tres procesos básicos: percepciónadquisición, almacenamiento y recuperación-utilización de la información (Jáuregui y Razumiejczyk, 2011; Naranjo y Gómez, 2015). Partiendo de esta visión algunas definiciones iniciales entendían las estrategias de aprendizaje como conductas o pensamientos puestos en práctica por un alumno para generar una secuencia de procesamiento de la información, su óptima integración y posterior recuperación, o en palabras de Dansereau (1985:120) "un conjunto de procesos o pasos que pueden facilitar la adquisición, almacenamiento y utilización de la información".

Una definición más integradora desde el ámbito de la Psicología de la Educación y la Psicopedagogía entiende las estrategias de aprendizaje como "comportamientos planificados que seleccionan y organizan mecanismos cognitivos, afectivos $y$ motóricos con el fin de enfrentarse a situaciones-problema, globales o específicas, de aprendizaje" (Monereo, 1990:4).

En relación específicamente con el aprendizaje de una segunda lengua, básicamente se ha aplicado la conceptualización psicológica anterior a la adquisición específica de esta competencia. Así, algunos autores han definido las estrategias de aprendizaje como "acciones, comportamientos o técnicas específicas que los estudiantes utilizan, a menudo intencionadamente, para mejorar sus progresos en aprender, asimilar y usar la segunda lengua" (Oxford, 1993, p. 18). La misma autora (2001, p. 166) de forma más específica entiende las estrategias de aprendizaje en general como aquellas "Operaciones empleadas por el alumno para ayudar a la adquisición, almacenamiento, recuperación y uso de información, acción específica tomada por el alumno para hacer el aprendizaje más fácil, rápido, más agradable, más auto dirigido, más eficaz y más transferible a situaciones nuevas". 
Son elementos comúnmente integrados en casi todas las definiciones, los siguientes (Cohen y Macaro, 2007; García Herrero, 2012a; Gargallo, 1995):

- En primer lugar se reconoce que las estrategias de aprendizaje son habilidades, competencias cognitivas y comportamientos concretos que los individuos ponen en funcionamiento de cara a un aprendizaje específico.

- Como habilidades que son, pueden ser identificadas (incluso por el propio sujeto que las utiliza) descritas, documentadas, aprendidas, enseñadas, influenciadas y perfeccionadas.

- Implican la integración e interacción mutua entre procesos y destrezas, entre las demandas que solicita una situación de aprendizaje y las competencias con que un sujeto se enfrenta a ella.

- Están vinculadas con aprendizajes específicos, sus contenidos, objetivos, etc., de ahí que tengan que ser cambiantes, dinámicas y en definitiva, flexibles.

- Están afectadas por variables múltiples:

- Varían en función de factores internos del sujeto, como por ejemplo su nivel de experiencia, de conocimientos en el área de aprendizaje, su implicación activa, competencia, actitud y aptitud, motivación, variables afectivas, emocionales, los gustos personales, las expectativas, etc.

- Cambian en función de aspectos externos al individuo, tales como las demandas de la propia situación de aprendizaje, metodología, actividades y sistemas de evaluación propuestos e implicados, condiciones y variables de la situación de enseñanza-aprendizaje, etc.

- Están dirigidas hacia un fin específico y permiten un aprendizaje con éxito y con mayor grado de eficacia y eficiencia. Por eso se entiende que su uso efectivo ha de ser deliberado, consciente, planificado y controlado.

- Implican un autoconocimiento de capacidades, potencial y aptitudes por parte del propio sujeto, autocontrol en su funcionamiento y capacidad de autoevaluación tanto del proceso de utilización como de los resultados obtenidos.

El uso eficiente, variado y habitual de estrategias de aprendizaje se ha asociado con mejores resultados académicos, jugando además en esa relación un papel importante el autoconcepto (Barca, Peralbo, Porto, Barca, Santorum y Castro, 2013).

Algunos estudios han sondeado otros aspectos psico-educativos que pueden estar asociados al funcionamiento de las estrategias de aprendizaje. Así por ejemplo, algunas tipologías de autoestima como por ejemplo la familiar y la académica, se asocian positivamente con niveles más elevados de uso de algunas estrategias de recuperación de información (Gázquez, Pérez, Ruiz, Miras y Vicente, 2006).

Un uso superficial de estrategias de aprendizaje se asocia, además de a bajos resultados académicos, a una mayor presencia de ansiedad a los exámenes y otras alteraciones emocionales, relación en la que parecen jugar un papel relevante aspectos como la confianza en sí mismo, la concentración o hábitos como dormir menos la noche antes de un examen (Fernández-Castillo, 2009; Fernández-Castillo y Caurcel, 2014; Furlan, Sánchez, Heredia, Piemontesi y Illbele, 2009).

Por último hacer alguna referencia también a que por lo que concierne a posibles diferencias de género, algunos autores han encontrado que las mujeres utilizan más estrategias que los hombres, tanto en general como en el aprendizaje específico de una segunda lengua (García Herrero, 2012b; Gázquez et al., 2006). 
Si el análisis de todas estas variables ha sido denso en los últimos años, no han sido menos los esfuerzos por desarrollar taxonomías de estrategias. En este ámbito nos centraremos a continuación.

\section{Clasificaciones y tipologías}

Desde hace ya bastante tiempo autores considerados hoy en día clásicos en el ámbito de conocimiento de la Psicología y la Psicología de la Educación se han propuesto adicionalmente el estudio de tipologías y posibles clasificaciones de estrategias de aprendizaje. Este abordaje se corresponde con aproximaciones comentadas más atrás derivadas del estudio del funcionamiento cognitivo humano y de los modelos originales del procesamiento de la información o de los procesos cognitivos básicos implicados en cualquier aprendizaje (Beltrán 1993). Puesto que esa aproximación se centra en procesos cognitivos tales como percepción, almacenamiento y recuperación-utilización de la información, algunas clasificaciones se suelen adscribir a esta perspectiva o posicionamiento.

Vamos a seguir este enfoque que puede resultar genérico para cualquier ámbito de aprendizaje pero concentrando nuestro campo de atención en el aprendizaje de una segunda lengua.

Así, mientras una primera diferenciación de estrategias de aprendizaje es la que distingue entre tres tipos básicos de ellas: cognitivas, metacognitivas y sociales (O’Malley y Chamot, 1990), otros optan por una clasificación más compleja que parte de discriminar entre estrategias primarias y secundarias (Weinstein y Mayer, 1986) o lo que otros autores han denominado estrategias directas e indirectas (Oxford, 1990; Rubin, 1975).

Seguiremos en este trabajo estas últimas tipologías clasificatorias dicotómicas, por ser más inclusivas, como se aprecia en la figura 1.

Desde estas aproximaciones se consideran como primarias a aquellas estrategias que favorecen de manera directa al aprendizaje, en nuestro caso el de una L2. Mientras que se considerarían estrategias secundarias o indirectas aquellas que aportan un apoyo diferido a dicho aprendizaje.

Siguiendo los planteamientos derivados de la Psicología cognitiva comentados, las estrategias primarias se establecen en base a un trabajo directo del sujeto con la información base de un aprendizaje, es decir serían aquellas que, sintéticamente, se relacionan con la recepción, memorización o almacenamiento y recuperación de información. Por consiguiente las primarias normalmente incluyen estrategias cognitivas y metacognitivas (Jiménez y Puente, 2014; Meichenbaum y Biemiller, 1992) mientras que las secundarias consideran tipologías como las personales, socioafectivas, ambientales, temporales, etc. 
Figura 1.- Tipologías de estrategias de aprendizaje

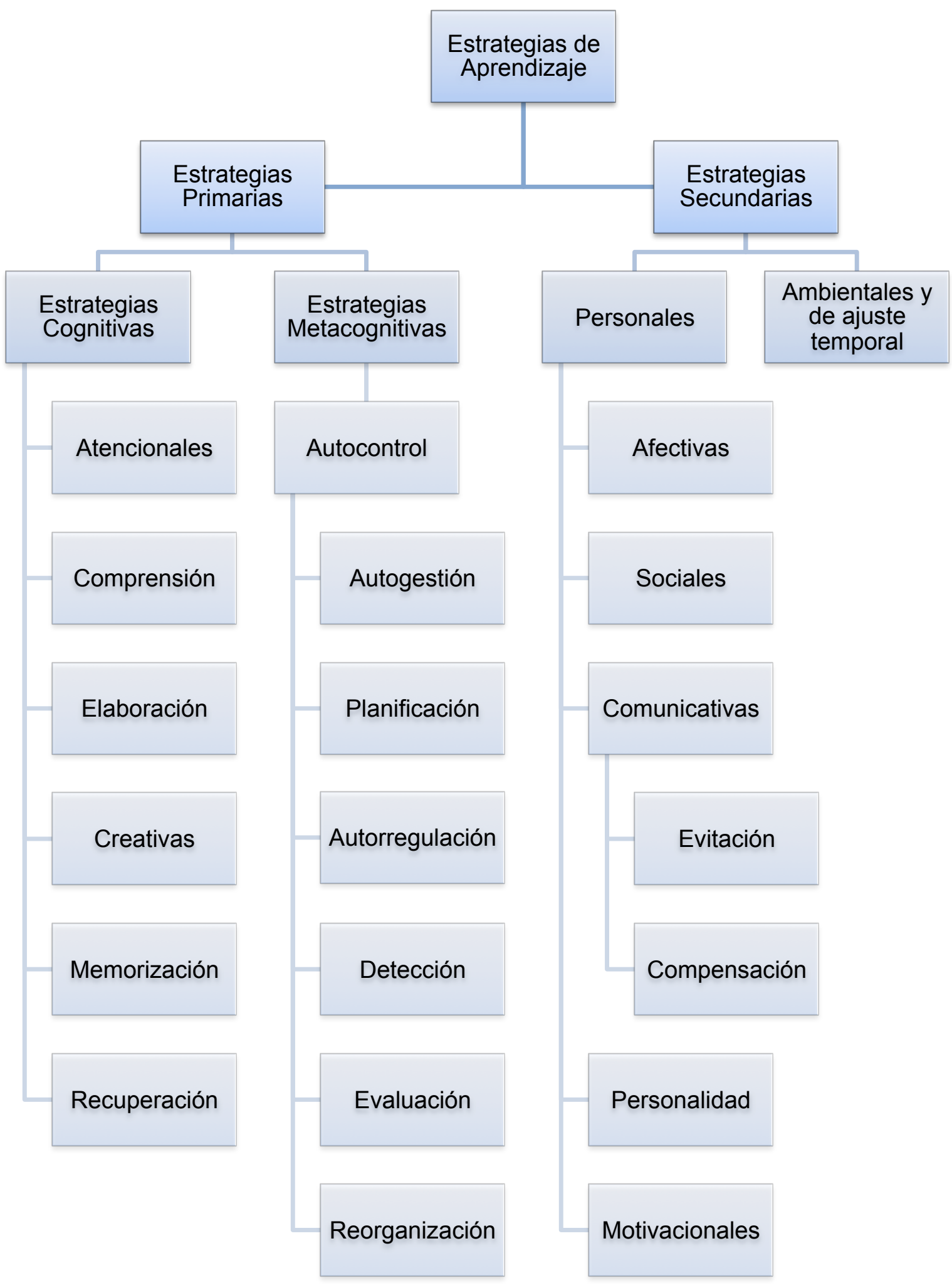




\section{1.- Estrategias primarias}

a) Las estrategias cognitivas implican la interacción entre el sistema cognitivo del sujeto y la información con la que se enfrenta (Hernández e Izquierdo, 2016; Jiménez y Puente, 2014). Esta interacción supone justamente el procesamiento de dicha información. Han sido consideradas como estrategias cognitivas primarias las siguientes:

- Estrategias atencionales, que permitirán el acceso a información relevante, en ellas se incluyen la atención general, atención selectiva, atención sostenida, etc.

- Estrategias de comprensión o de adquisición, implicando frecuentemente un esfuerzo de apertura a la integración de nuevos elementos en el aprendizaje de una segunda lengua (García Herrero, 2013a y 2013b). Son ejemplos en esta categoría las tácticas de razonamiento, estructuración de la información, elaboración de esquemas y resúmenes, análisis de contenidos, etc. Igualmente la utilización de sinónimos, asociación, recursos gestuales, establecimiento de un significado en función de información contextual, ubicación contextualizada de palabras nuevas, etc.

- Estrategias de elaboración, codificación, transformación y organización del contenido. Suponen un trabajo con contenidos de aprendizaje para hacerlos más accesibles, inteligibles, etc., conectando en cierta medida los novedosos con aquellos que ya se habían adquirido o que se dominan. Permiten que los contenidos sean comprensibles, favorecerán la retención, el posterior análisis y potencian los resultados del aprendizaje tales como transformaciones, generalizaciones y aplicaciones y puesta en contexto de lo aprendido. Son ejemplos de ello la ordenación de contenidos en secuencias, la taxonomización, el establecimienteo de jerarquías, los agrupamientos, etc.

- Estrategias creativas o de individualización. En ellas se incluyen aspectos como la innovación en la elaboración y en el procesamiento, el pensamiento divergente sobre la información procesada, etc.

- Estrategias de memorización. En estas se incluyen estrategias que favorecen el almacenamiento o retención de una determinada información. Algunos autores han categorizado la memorización como una estrategia independiente de las cognitivas (García Herrero y Jiménez Vivas, 2014; Oxford, 1990), aunque desde un punto de vista psico-educativo, es más apropiado considerar el uso de estrategias de memorización como un aspecto básico del funcionamiento cognitivo humano. Son ejemplos de ellas la repetición, la copia, el subrayado, el repaso, la reproducción de ejercicios y prácticas, etc.

- Estrategias de recuperación o acceso al material almacenado, incluyendo aquí estrategias de búsqueda dirigida, y procesos como evocación, reconocimiento, etc.

b) Las estrategias metacognitivas implican un trabajo algo más indirecto con la información, aunque siguen siendo directamente facilitadoras de aprendizaje. De hecho, son las más frecuentemente utilizadas por los estudiantes de todos los niveles de competencias lingüísticas (García Herrero y Jiménez Vivas, 2014). Suponen que el propio sujeto sea consciente del proceso de aprendizaje en sí, un cierto grado de autoconocimiento y una reflexión sobre el transcurso de su trabajo (Hernández e Izquierdo, 2016; Jiménez y Puente, 2014). Suelen dominarse por individuos con experiencia de aprendizaje y no tanto por principiantes y como aspecto general a todos ellos se encontraría en mayor o menor medida el autocontrol de los propias dimensiones cognitivas implicadas en el proceso. Asume que el sujeto sea consciente de los procesos, tenga una idea más o menos clara de cómo funcionan en su caso 
particular y pueda gestionar su funcionamiento. Algunos de las estrategias más importantes consideradas en esta categoría serían, entre otras:

- Autogestión. Es una de las estrategias básicas en la medida en que implica la aptitud de controlar el funcionamiento del aprendizaje propio. Implica un funcionamiento autónomo del individuo y el aprovechamiento del potencial y las posibilidades disponibles. Se incluyen aquí a organización general de aspectos como el tiempo, los pasos para alcanzar una meta específica, la calendarización, etc.

- Planificación o anticipación. Implica un proceso de previsión, antelación y de adelanto con la suficiente perspectiva, de los procesos y acontecimientos que se ubican en el futuro. Son ejemplo de ello conocer y trabajar en habilidades y conocimientos y en definitiva los aspectos, circunstancias y contenidos que son requisito previo para aprendizajes posteriores.

- Autorregulación. Supone un cierto control y seguimiento de los avances que han de llevarse a cabo para alcanzar un objetivo planificado. Implica llevar a cabo pasos específicos, atendiendo a realizarlos de manera adecuada, motivada y corrigiendo posibles dificultades durante el proceso.

- Detección de problemas. Altamente relacionada con el punto anterior, en este caso estamos ante una estrategia que puede ser considerada como independiente y complementaria. Se establece en torno a la habilidad de discriminar, incluso con antelación suficiente, problemas y complicaciones que surgen durante la tarea de aprendizaje. O bien para resolverla cuando se ha producido o bien para poner en marcha tareas que impidan que esa traba, limitación u obstáculo llegue a producirse.

- Evaluación y autoevaluación. Implica la pericia para darse cuenta tanto de fallos y complicaciones como de aciertos y éxitos, pasados, presentes y futuros, de cara al aprovechamiento de esa información en el trabajo de aprendizaje futuro. Se diferencia de la detección de problemas en que la evaluación es un proceso más detenido, profundo y que se lleva a cabo tras cierto tiempo y en ocasiones con una visión general o de conjunto. Los buenos estudiantes de una segunda lengua, suelen saber donde son buenos y donde no lo son tanto.

- Reorganización. Puede ser entendida como un proceso de toma de decisiones tras un proceso de evaluación, en la medida en que implica decidir qué aspectos han de cambiarse a tenor de la experiencia previa y el resultado de su valoración y evaluación sincera. Implica una ubicación previa por parte del propio sujeto sobre su lugar en el proceso y la dinámica de aprendizaje. De esta manera por ejemplo se decidirá cambiar aspectos, modificar procesos, reemplazarlos por otros o incluso alterar el orden y la inclusión o no de los objetivos, actividades, metodología, etc., en el proceso de enseñanzaaprendizaje.

\section{2.- Estrategias secundarias, indirectas o de apoyo}

Se trata de tácticas que facilitan condiciones óptimas para que las estrategias primarias permitan alcanzar el mejor resultado posible (Oxford, 2001). Un sistema bastante integrador de este segundo conjunto general podría diferenciar entre estrategias: a) personales $\mathrm{o}$ individuales y b) ambientales y de ajuste temporal. A continuación se analizarán con mayor detalle ambos conjuntos.

a) Personales o individuales. Se incluirían en esta categoría estrategias particulares de cada individuo en cuestión y por tanto bastante personalizadas y que operan como 
puntos fuertes o aspectos facilitadores del funcionamiento de las estrategias primarias. Las más importantes son las siguientes:

- Estrategias afectivas. Se relacionan con tácticas que ponen en marcha algunos individuos para identificar, controlar y potenciar las emociones y los sentimientos propios, de cara a favorecer el proceso de aprendizaje. Un déficit en estas estrategias puede incrementar aspectos como la frustración o la alteración emocional (ansiedad, estrés, etc.), durante el aprendizaje de una L2 y sobre todo en actividades concretas implicadas tales como hablar en público, exposiciones orales, etc.

- Estrategias sociales. Estas implican la interacción con los demás y la forma que incide en el proceso de aprendizaje. Aspectos incluidos aquí serían por ejemplo la asertividad, las habilidades sociales, la capacidad y destreza para interactuar con la red social de apoyo, etc. Sus implicaciones van más allá de las relaciones sociales en sí, pues pueden tener repercusiones sobre por ejemplo, procesos cooperativos de aprendizaje, el trabajo en grupos, la relación espontánea y la inserción en contextos específicos de aprendizaje.

- Estrategias comunicativas. Permiten potenciar el uso de los productos del aprendizaje en un contexto social, en el caso específico de la lengua implican, entre otros procesos, su puesta en funcionamiento. Estas estrategias son especialmente relevantes en el caso del aprendizaje de una $L 2$ ya que suponen la potenciación a partir de la práctica y la implementación y por tanto se consideran habitualmente como estrategias indirectas. Han de ser entendidas como aspectos independientes de las incluidas en el punto anterior.

Cuando no se ponen en funcionamiento de manera adaptativa, suelen ser sustituidas por dos tendencias menos eficientes, e incluso considerada la primera de ellas como opción comportamental desadaptativa cuando conduce a comportamientos de evitación. Las estrategias a las que nos referimos son las conductas de afrontamiento de la evitación y las estrategias de compensación (O’Malley y Chamot, 1990).

- Los estudiantes más avanzados suelen poner en marcha habilidades para soslayar o afrontar la tendencia a generar procesos de evitación. Los comportamientos de evitación implican que un sujeto tienda a no enfrentarse con aquellos aspectos del aprendizaje que, estando mediatizados por una experiencia de interacción social o comunicativa, no domina, desconoce o que le generan alteración emocional como por ejemplo ansiedad. La comunicación se reduce en estas circunstancias, tiende a ser empobrecida contribuyendo al aislamiento o a la minimización de interacciones sociales, sobre todo cuando las habilidades y competencias de comunicación no son demasiado avanzadas, o las circunstancias se perciben como amenazantes, percepción de inferioridad y baja autoestima, etc.

- Las estrategias de compensación suponen que un individuo intentará encontrar alternativas para determinadas tareas o estructuras que no domina o desconoce. Si las estrategias de comunicación son efectivas, permitirán un uso de la compensación más eficiente. Son ejemplos bastante habituales de estas destrezas en el aprendizaje de una L2 el uso de circunloquios, la búsqueda de sinónimos, de incitación a que hable el interlocutor, preguntas a éste, etc. A diferencia de la opción anterior, el sujeto hace frente a la situación de comunicación, quizá no de forma totalmente eficiente, pero en cualquier caso no evitándola. 
- Personalidad. Ciertas estrategias relacionadas con características personales que ponen en marcha algunos individuos se pondrían incluir en este apartado. Se basan en aprovechar o potenciar rasgos y particularidades individuales que pueden contribuir a la adquisición de la L2. Ejemplos de ellas serían la utilización de la extroversión, la simpatía, afabilidad y amabilidad, responsabilidad, apertura mental y otros factores de personalidad que potencian o dificultan las relaciones personales, tareas específicas de aprendizaje, los sistemas de evaluación, etc. (que pueden relacionarse con que un alumno intervenga más en clase, que pregunte, que interactúe con extraños en el aprendizaje de una L2, etc.). También pueden ser incluidos en este apartado aspectos como el locus de control, la atribución de causalidad y otras características de la persona, relativamente estables o permanentes en el tiempo y frente a diferentes situaciones que incidirían en el proceso de enseñanza-aprendizaje de la L2.

- Estrategias motivacionales. Un elemento importante que suele incluirse en este apartado son aquellas estrategias que ponen en marcha algunos individuos dirigidas a incrementar su propia motivación, la potenciación de las actitudes positivas hacia el aprendizaje y otras estrategias de sensibilización (Beltrán, 1993). Los buenos estudiantes de idiomas implementan con frecuencia estrategias dirigidas a, por ejemplo, el incentivo de la motivación intrínseca, la puesta en marcha a sistemas de autorrefuerzo, incremento de la curiosidad, la búsqueda activa de elementos motivadores personalizados, la potenciación de la autoeficacia, etc.

b) Estrategias ambientales y de ajuste temporal. En este apartado se incluirían aquellas tácticas que usan los estudiantes para mejorar su rendimiento y que conciernen a ajustes contextuales, espaciales, estimulares, etc. Son ejemplo de ello el ambiente de estudio y trabajo, el lugar específico, la distribución de materiales, la iluminación, eliminación o control de elementos distractores, etc., por citar algunos.

Por lo que concierne a estrategias temporales, se consideran como tales la organización del aprendizaje en relación con el tiempo, siendo ejemplos de ello la calendarización y estructuración del trabajo en base a horarios, etc.

En el caso del aprendizaje de una L2, algunos sujetos planifican estructuras de trabajo, repaso, etc., usando tiempo libre, visionado de materiales audiovisuales y películas en el idioma de L2, favoreciendo ámbitos de interacción con hablantes nativos, todo ello con independencia de horarios y estructuras de aprendizaje en cursos y enseñanzas regladas. De todas las estrategias de aprendizaje consideradas, parece que las más utilizadas en aprendizajes reglados son las metacognitivas, las afectivas, las comunicativas y de compensación seguidas de las cognitivas. Más específicamente, se ha señalado que en el aprendizaje de una L2 y a un nivel universitario, las estrategias más utilizadas son el análisis, el repaso de reglas gramaticales, la división de palabras y frases, las estrategias comunicativas y la utilización de analogías y contrastes (García Herrero, 2013b).

Para concluir este apartado, recordar que todos los individuos que se enfrentan con un proceso de aprendizaje y adquisición de una L2 usan y ponen en marcha estrategias de aprendizaje, incluso no siendo en ocasiones conscientes de ello. El conocimiento de las mismas permite su optimización, la eficiencia durante el proceso y marcará diferencias entre estudiantes eficientes y poco eficientes y por consiguiente el acceso a mejores resultados. De ahí que sea necesario abordar a continuación un apartado centrado en posibilidades de atención a estas cuestiones. 


\section{Consideraciones aplicadas programas de intervención y optimización de estrategias de aprendizaje}

Según diversos estudios, los estudiantes de mayor y de menor éxito académico coinciden en utilizar estrategias de aprendizaje bastante similares, encontrándose las diferencias entre ambos conjuntos de estudiantes más en la efectividad del uso que hacen de dichas estrategias que en el tipo de ellas (García Herrero y Jiménez Vivas, 2014; Liu, 2004). A pesar de esto, se ha encontrado evidencia de algunas estrategias que parecen predominar y usarse más eficazmente por alumnos con buenos resultados en aprendizaje de L2. Parece por ejemplo que los buenos alumnos intentan encontrar en mayor grado semejanzas de la L2 con la lengua materna, lo que se asocia a un aprendizaje más efectivo (García Herrero y Jiménez Vivas, 2014). Estos mismos autores encontraron evidencia de que entre las estrategias más utilizadas en el aprendizaje de una L2 se incluían, además de las tácticas metacognitivas, el repaso y el control de la ansiedad ante situaciones de interacción usando la L2.

Diferentes autores han considerado o planteado que los sistemas de intervención o entrenamiento de estrategias de aprendizaje han de incluir al menos los siguientes aspectos:

a) Iniciativas dirigidas a que el alumno sea consciente de las propias estrategias que pone en funcionamiento en procesos de aprendizaje (Monereo, 1992). En este punto suelen incluirse técnicas como la autoobservación y registro de comportamientos y procesos cognitivos puestos en funcionamiento, un autoanálisis de estrategias con la descripción de las mismas y una exposición de la eficiencia observada para cada una de estas estrategias. En general estas técnicas van dirigidas a que este autoconocimiento permita potenciar tanto la organización previa de la tarea y de lo que se va a hacer, como del seguimiento, la organización y evaluación de la misma.

Estas técnicas de autoconocimiento pueden ser potenciadas si se someten a valoración, tanto del propio sujeto como externa. Por ejemplo si se explican en grupos de discusión, ante expertos o docentes y se le ofrece una retroalimentación y opiniones al sujeto.

b) Ampliar el conocimiento de estrategias tanto en un sentido general, como de las utilizadas por compañeros, propuestas por profesionales de las ciencias de la educación u otras personas que se enfrentan a aprendizajes similares. Dentro de esta categoría de técnicas destaca especialmente el aprendizaje por observación de las estrategias puestas en marcha por otros y la imitación (Beltrán, 1993; Monereo, 1994).

Por supuesto que llegar a conocer las estrategias de aprendizaje que mejor se aplican y mejores resultados permiten alcanzar a cada sujeto, incluye la instrucción directa o la enseñanza en sí de las técnicas y estrategias específicas. Son especialmente utilizadas estas iniciativas en el seno de seminarios sobre técnicas de estudio o cursillos formativos para el desarrollo de competencias, por citar algunos ejemplos.

c) Fomento de la motivación y la automotivación. Para fomentarla en relación con la adquisición de nuevas estrategias de aprendizaje, los alumnos han de valorarlas, otorgarles utilidad y estar dispuestos a trabajar para dominarlas.

d) Método de autoinstrucciones (Meichenbaum y Biemiller, 1992). Básicamente consiste en que el sujeto va verbalizando las tareas que desarrolla o se propone llevar a cabo. Estas técnicas fomentan la autorregulación y generan que los individuos se 
mantengan en una línea de trabajo, que sean conscientes de fallos, errores y aspectos a mejorar y además suelen aumentar la automotivación.

e) Paralelamente al punto anterior, hemos de tener en cuenta que el perfeccionamiento en el uso de estrategias se alcanza mejor y antes si el sujeto cuenta con una buena, valiosa y sincera fuente de retroalimentación. Así el estudiante podrá darse cuenta antes de qué puede estar fallando o qué va mal, conocer de manera eficaz por qué está teniendo dificultades y establecer qué ha de hacer para corregir o evitar ese error. A menudo estos planteamientos se incluyen deliberadamente en procesos paralelos a la guía docente que acompaña a la puesta en práctica de las estrategias en el seno de programas de entrenamiento.

f) En el caso de las estrategias para el aprendizaje de una L2, cobra importancia la influencia del contexto social y lingüístico en la medida en que algunas estrategias se corresponderán con él y el aprendizaje de dichas tácticas habrá de ponerse en marcha en contextos naturales donde las estrategias y los procesos de aprendizaje en sí, se pondrán posteriormente en funcionamiento contextualizado.

g) Por último, se deberán implementar sistemas que permitan conocer si un sujeto está usando de manera adecuada las estrategias de aprendizaje, es decir, habrá de considerarse algún sistema de evaluación y valoración final. A partir de cuyos resultados se podrá decidir si mantener la intervención o no, si modificarla, cambiarla en cuantos a sus objetivos, actividades, ejercicios, etc.

Cualquier técnica de intervención que se vaya a instruir ha de ser contextualizada, relacionada con los objetivos de aprendizaje, el potencial y la actitud del alumno y personalizada a sus características y las del proceso y el contexto en sí.

\section{Conclusiones}

Diversos ámbitos de conocimiento e investigación, entre los que se encuentra la Psicología de la Educación, vienen centrando su atención en variables y procesos que inciden en que una segunda lengua llegue a ser aprendida de manera óptima y eficiente. Este campo ha generado gran cúmulo de conocimientos y teorías, pero por ahora todavía no ha respondido con rotundidad a multitud de cuestionamientos e interrogantes.

El propio proceso de adquisición de una lengua todavía plantea profundos ámbitos de desconocimiento. De hecho, aunque los individuos parecen adquirir la lengua materna incorporado elementos gramaticales sin necesidad de una programación estructurada e instruccional, sin una aparente planificación prediseñada y a lo largo de un proceso interactivo eminentemente natural de asimilación semántico-funcional, no parece ocurrir lo mismo cuando se enfrentan a procesos de adquisición de una segunda lengua.

En este segundo caso, aunque parece claro que las estrategias y los estilos de aprendizaje que los individuos ponen en funcionamiento desempeñan un papel relevante en el proceso de enseñanza-adquisición, sigue siendo todavía mucho lo que queda por conocer. Sobre este punto nos hemos detenido en este trabajo, intentando llevar a cabo un análisis conceptual y descriptivo sobre estos aspectos.

Los procesos que diferencian a los estudiantes buenos de los que no lo son tanto, han merecido la atención de los investigadores desde hace tiempo. Posiblemente las diferencias entre ellos no se deban tanto, como hemos visto, a las estrategias 
utilizadas en sí, sino más a estilos personales de aprendizaje o a la forma de reflexionar sobre el propio proceso de avance, corrección y retroalimentación derivada de éxitos y fracasos personales.

El entrenamiento específico de estrategias de aprendizaje se asocia, como no podía ser de otra manera a que los estudiantes las incorporen de forma efectiva y eficiente a su sistema de trabajo a lo largo de la adquisición de la L2, a un mayor uso social, e incluso en contextos naturales. Los estudiantes exitosos suelen generar nuevas estrategias y diversifican y adaptan a su aptitudes personales el uso de las estrategias entrenadas y que les funcionan (Pikabea, Joaristi y Lizasoain, 2004), lo que en el fondo denota un autoconocimiento efectivo.

Por todo ello, parece evidente que hemos de asumir un grado de complejidad elevado en éste ámbito de conocimiento que implica redoblar nuestros esfuerzos futuros. Y aunque por ahora podemos seguir trabajando de cara a potenciar la eficacia en el aprendizaje de idiomas, habremos de continuar haciendo esfuerzos por sintonizar lo que sabemos hasta ahora y lo que es imprescindible saber.

\section{Referencias}

Barca, A., Peralbo, M., Porto, A. M., Barca, E. y Santorum, R. y Castro, F. V. (2013). Estrategias de aprendizaje, autoconcepto y rendimiento académico en la adolescencia. Revista Galego-Portuguesa de Psicoloxía e Educación, 21, 195-212.

Beltrán, J. (1993). Procesos, estrategias y técnicas de aprendizaje. Madrid: Síntesis.

Castejón, J.L. (2014). Aprendizaje y rendimiento académico. Alicante: Editorial Club Universitario.

Chamot, A., O'Malley, M., Kupper, L., y Impink-Hernandez, M. (1988). A Study of Learning Strategies in Foreign Language Instruction: Findings of the Longitudinal Study. McLean, Va.: Interstate Research Associates.

Cohen, A. y Macaro, E. (2007). Language learner strategies. Oxford: Oxford University Press.

Dansereau, D. F. (1985). Learning strategy research. En J. V. Segal, S. F. Chipman y R. Glaser (Eds.). Thinking and learning skills (Pp. 209-239). Vol. 1. Hillsdale: Erlbaum.

Fernández-Castillo, A. (2009). Ansiedad durante pruebas de evaluación académica: influencia de la cantidad de sueño y la agresividad. Salud Mental, 32, 479-486.

Fernández-Castillo, A. y Caurcel, M. J. (2014). State test-anxiety, selective attention and concentration in university students. International Journal of Psychology, 50, 265-271.

Furlan, L. A., Sánchez, J., Heredia, D., Piemontesi, S. y Illbele, A. (2009). Estrategias de aprendizaje y ansiedad ante los exámenes en estudiantes universitarios. Pensamiento Psicológico, 5, 117-124.

García Herrero, M. M. (2012a). Combinaciones de estrategias en el aprendizaje de la lengua extranjera. Revista Galego-Portuguesa de Psicoloxía e Educación, 20, 133-153.

García Herrero, M. M. (2012b). Diferencias en el uso de Estrategias en el Aprendizaje de la lengua Extranjera según el género. Docencia e Investigación, 37, 61-80.

García Herrero, M. M. (2013a). Análisis de la utilización de estrategias en el aprendizaje de la lengua extranjera. Revista de Investigación Educativa, 31(1), 53-76.

García Herrero, M. M. (2013b). Utilización de estrategias y selección de estilos por estudiantes universitarios, ya titulados, en el aprendizaje de la lengua extranjera. Educar, 49, 127-151.

García Herrero, M. M. y Jiménez Vivas, A. (2014). Estrategias en el aprendizaje de la lengua extranjera y niveles de competencia en estudiantes universitarios de magisterio. Revista de Investigación Educativa, 32, 363-378. 
Gargallo, B. (1995). Estrategias de aprendizaje. Estado de la cuestión. Propuestas para la intervención educativa. Teoría de la educación. Revista Interuniversitaria, 7(1), 53-75.

Gázquez, J. J., Pérez, M. C., Ruiz, M. I., Miras, F. y Vicente, F. (2006). Estrategias de aprendizaje en estudiantes de enseñanza secundaria obligatoria y su relación con la autoestima. International Journal of Psychology and Psychological Therapy, 6, 51-62.

Griffiths, C. (2008). Strategies and good language learners. En C. Griffiths (Ed.). Lessons from Good Language Learners (Pp. 83-98). New York: Cambridge University Press.

Hernández, J. e Izquierdo, J. (2016). Metacognición y comprensión oral en L2. Observación de la práctica docente en nivel universitario. Revista Electrónica de Investigación Educativa, 18(1), 39-52.

Jáuregui, M. y Razumiejczyk, E. (2011). Memoria y aprendizaje: una revisión de los aportes cognitivos. Psicología y Psicopedagogía, 26, 20-44.

Jiménez, V. y Puente, A. (2014). Modelo de estrategias metacognitivas. Revista de Investigación Universitaria, 3, 1116.

Liu, D. (2004). EFL proficiency, gender and language learning strategy use among a group of Chinese technological institute English majors. ARECLS E Journals, 1, 20-28.

Meichenbaum, D. y Biemiller, A. (1992). In search of student expertise in the classroom: a metacognitive analysis. En M. Pressley, K.R. Harris y J.T. Guthrie (Eds.). Promoting academic competence and literacy in school (pp. 356). San Diego, CA: Academic Press.

Monereo, C. (1990). Las estrategias de aprendizaje en la educación formal: enseñar a pensar y sobre el pensar. Infancia y aprendizaje, 13(50), 3-25.

Monereo, C. (1992). Estrategias de aprendizaje y enseñanza. Madrid: Pascal.

Monereo, C. (1994). Estrategias de enseñanza y aprendizaje. Barcelona: GAÓ.

Naranjo, Y. y Gómez, S. A. (2015). Estado del arte de los modelos de niveles de procesamiento de la información. Consultado en: http://hdl.handle.net/10819/2596

O'Malley, J. M. y Chamot, A. U. (1990). Learning Strategies in Second Language Acquisition. Cambridge: Cambridge University Press.

Oxford, R. L. (1990). Language learning strategies: What every teacher should know. New York: Newbury House.

Oxford, R. L. (1993). Language learning strategies in a nutshell: Update and ESL suggestions. TESOL Journal, 2(2), 1822.

Oxford, R. L. (2001). Language learning strategies. En R. Carter y D. Nunan (Eds.). Teaching English to speakers of other languages (Pp. 166-72). Cambridge: Cambridge University Press.

Pikabea, I., Joaristi, L. y Lizasoain, L. (2004). Uso social de un segundo idioma y estrategias de aprendizaje. Revista de Investigación Educativa, 22, 459-472.

Rodríguez, S., Valle, A., Nuñez, J. C. (2014). Enseñar a aprender: estrategias, actividades y recursos instruccionales. Madrid: Pirámide.

Rubin, J. (1975). What the "good language learner" can teach us. TESOL Quarterly, 9(1), 41-51.

Takeuchi, O. (2003). What can we learn from good language learners: A qualitative study in the Japanese foreign language context. System, 31, 385-392.

Weinstein, C. E. y Mayer, R. F. (1986). The teaching of learning strategies. En M.C. Wittrock (Ed.). Handbook of research on teaching (Pp. 315-327). New York: McMillan.

Wu, Y. L. (2008). Language learning strategies used by students at different proficiency levels. Asian EFL Journal, 4 (10), 75-95. 